\title{
Combined Longitudinal and Lateral Control Design for String Stable Vehicle Platooning within a Designated Lane
}

\author{
Roozbeh Kianfar *, Mohammad Ali**, Paolo Falcone *, Jonas Fredriksson *
}

\begin{abstract}
We propose a combined longitudinal and lateral control approach for vehicle platooning within a designated lane. We combine linear frequency and time domain methods, to design longitudinal control that ensures string stability while enforcing safety, comfort and actuator limitations. In addition, we design lateral control that accounts for speed variations induced by the longitudinal control as well as safety, comfort and actuator limitations using convex optimization methods.
\end{abstract}

\section{INTRODUCTION}

In a vehicle platoon, a chain of vehicles follow a lead vehicle, preferably driven by a professional driver, automatically. By reducing the driving automation problem to a vehicle following problem, benefits associated with automated driving can be obtained at low cost. While research efforts to enable platooning date back to the eighties [10], recent advances in sensing, computational and communication capabilities have made the deployment of platooning technology in commercial vehicles more realistic than ever [2].

To enable vehicle platooning within a designated lane, control of both the longitudinal and the lateral motion of the vehicle is required. The primary objective of the longitudinal control is to regulate the speed of individual vehicles to the desired platoon speed via the brakes and throttle. However, while regulating speed, it is also important to ensure the safety and string stability of the platoon. Since the feedback loops of the vehicles in the platoon are coupled, disturbances acting on one vehicle can propagate and influence other vehicles in the platoon. If such disturbances are always attenuated, the vehicle platoon is string stable.

A rigorous study on disturbance propagation in vehicle platoons is provided in [12]. In fact, it is shown in [12] that if vehicles use only relative spacing information, the platoon will be string unstable for any linear controller. In [9], a necessary and sufficient frequency domain condition is derived for string stability, requiring a communication link with the nearest preceding vehicle only. While there are many benefits with control design in the frequency domain, time domain constraints on the vehicle states and inputs are difficult to incorporate using frequency domain tools. In [3] and [5], the string stability requirement is translated into time domain inequality constraints and addressed within the Model Predictive Control (MPC) framework allowing for other requirements like safety and comfort constraints to be

\footnotetext{
* Department of Signals and Systems, Chalmers University of Technology, SE-412 96 Göteborg, Sweden, Email:roozbeh,jonas.fredriksson@chalmers.se

† Volvo Car Corporation, Active Safety \& Chassis, 96620/PV4A, 40531 Göteborg Sweden, E-mail: mohammad.ali@volvocars.com
}

incorporated as well [6], [4]. However, the methods proposed in [3] and [5] require that each vehicle broadcasts its intended trajectory, which might be impractical.

As for the lateral controller, its objective is to keep the vehicle within the lane through steering. It is well known that the combined longitudinal and lateral vehicle dynamics are non-linear and are thus challenging to simultaneously control with linear controllers. While optimization methods that enable the use of MPC for nonlinear systems exist, it is also well known that such methods are associated with problems of local optima.

In this work, we divide the control problem into subproblems and propose a control approach that,

1) ensures string stability while only requiring information about the preceding vehicle's acceleration,

2) abides longitudinal and lateral safety, comfort and actuator constraints and

3) maintains the vehicle within the designated lane, while accounting for predicted speed variations induced by the longitudinal control.

The sub-problems are all solved using convex optimization methods only, thus guaranteeing global optimality. The approach is detailed in the following sections.

\section{MODELING}

In this section, we present the vehicle model used in Section IV, as basis for the control design. Consider the notation introduced in Figure 11. We use the following set of differential equations to describe the inter-vehicle longitudinal dynamics and the vehicle motion within the lane, subject to the lateral and yaw dynamics,

$$
\begin{aligned}
m \dot{v}_{y} & =-m v_{x} \dot{\psi}+2\left[F_{y_{f}}+F_{y_{r}}\right] \\
J_{z} \ddot{\psi} & =2\left[l_{f} F_{y_{f}}-l_{r} F_{y_{r}}\right], \\
\dot{e}_{\psi} & =\dot{\psi}-\dot{\psi}_{d} \\
\dot{e}_{y} & =v_{y}+v_{x} e_{\psi} \\
\dot{v}_{x} & =a_{x} \\
\dot{e}_{p} & =e_{v}-a_{x} h \\
\dot{e}_{v} & =a_{x}^{\text {target }}-a_{x} \\
\dot{a}_{x} & =\frac{-1}{\tau} a_{x}+\frac{K}{\tau} a_{d e s},
\end{aligned}
$$

where $m$ and $J_{z}$ denote the vehicle mass and yaw inertia, respectively, $l_{f}$ and $l_{r}$ are the distances of the vehicle center of gravity from the front and rear axles, respectively, $v_{x}$ and $v_{y}$ are the longitudinal and lateral velocities, respectively, in the 


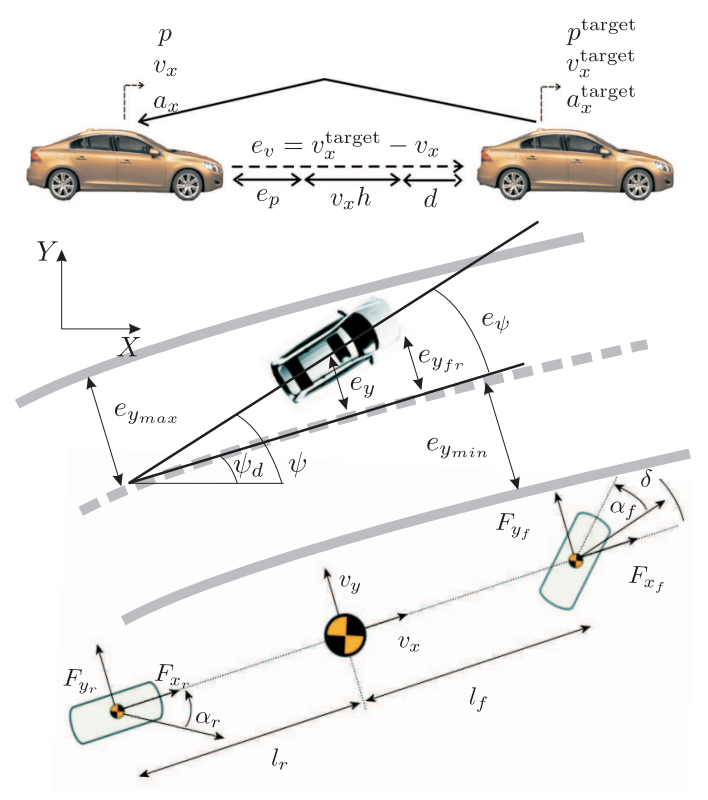

Fig. 1. Vehicle modeling notation.

vehicle body frame, $\dot{\psi}$ is the turning rate, where $\psi$ denotes the vehicle orientation w.r.t. the fixed global frame $(X, Y)$ in Figure 1. $F_{y_{f}}, F_{y_{r}}$ are the lateral tire forces at the front and rear axles, respectively. In (1c) and (1d), $e_{\psi}$ and $e_{y}$ denote the vehicle orientation and position errors, respectively, w.r.t. the road centerline and $\psi_{d}$ is the orientation of the road centerline.

The position error $e_{p}$ w.r.t. a desired distance from the preceding vehicle is defined as $e_{p}=p^{\text {target }}-p-d-v_{x} h$, where $d$ and $h$ are the standstill distance and the constant headway time, respectively, $e_{v}$ denotes the relative velocity between vehicles, i.e., $e_{v}=v_{x}^{\text {target }}-v_{x}$.

The lateral tire forces in (1a) and (1b) are approximated as linear functions of the slip angles,

$$
F_{y i}=-C_{i} \alpha_{i}, i \in\{f, r\},
$$

where $C_{i}$ are the slopes of the tire force characteristics and $\alpha_{f}, \alpha_{r}$ are tyre slip angles which, for small values, can be approximated as,

$$
\alpha_{f}=\frac{v_{y}+l_{f} \dot{\psi}}{v_{x}}-\delta, \alpha_{r}=\frac{v_{y}-l_{r} \dot{\psi}}{v_{x}},
$$

where $\delta$ denotes the front steering angle as depicted in Figure 1 .

\section{A. Longitudinal vehicle dynamics}

While the system (1) describes both the longitudinal and lateral vehicle dynamics, we note that the longitudinal inter vehicle dynamics can be described only considering the Equations (1f)- (1h). In (1f)- (1h) the longitudinal dynamics have been modeled as a system of connected double integrators augmented with a first order filter to account for actuator (engine/brake) dynamics. We compactly describe the inter vehicle longitudinal dynamics in state space form,

$$
\dot{\chi}(t)=A_{\chi} \chi(t)+B_{u} u(t)+E_{w} w(t),
$$

where $\chi=\left[\begin{array}{llll}v_{x} & e_{p} & e_{v} & a_{x}\end{array}\right]^{\mathrm{T}}, u=a_{\mathrm{des}}, w=a_{x}^{\text {target }}$, are the state, the control and the disturbance vectors, respectively. Notice that the acceleration of the preceding vehicle is considered an exogenous signal which, in a real time application, can be provided through a communication link. We also remark that even though Equations (1f)- (1h) are sufficient for describing the inter vehicle dynamics, we have included (1e) in the model (4). We motivate this design choice in Section IV-C

\section{B. Lateral vehicle dynamics}

We compactly rewrite Equations (1a)-(1d) and (2)-(3) to form the LPV model,

$$
\dot{\xi}(t)=A_{\xi}(\rho) \xi(t)+B_{u} u(t)+E_{w} w(t),
$$

where $\xi=\left[v_{y}, \dot{\psi}, e_{\psi}, e_{y}\right]^{T}, u=\delta, w=\dot{\psi}_{d}$ are the state, input and disturbance vectors, respectively, and $\rho=v_{x}$ is a parameter of the model $A_{\xi}$.

Clearly, we have excluded Equation (1e) from the model (5) and instead consider the longitudinal velocity $v_{x}$ as a model parameter. We motivate this in Section IV-C

\section{DESIGN REQUIREMENTS AND LIMITATIONS}

In this section we introduce requirements and limitations that the control design in Section IV needs to fulfil in order to achieve desired platoon behaviour.

\section{A. Frequency domain requirements (longitudinal)}

Formally, string stability can be defined with respect to different signals, see e.g. [9], [1]. Among alternative definitions of string stability, we adopt a predecessor-follower string stability criterion in this paper that is based on the $\mathcal{L}_{2}$ norm of the acceleration signals.

Definition 1: (String stability): A vehicle platoon is predecessor-follower string stable w.r.t acceleration disturbances if the following holds,

$$
\|\Gamma(j \omega)\|_{\infty}=\sup _{\omega \in \mathcal{R}}|\Gamma(j \omega)| \leq 1
$$

where $\Gamma(j \omega)$ denotes the transfer function from $a_{x}^{\text {target }} \rightarrow$ $a_{x}$ and $|$.$| denotes magnitude of the system \Gamma(j \omega)$. This condition can also be written in terms of the $\mathcal{L}_{2}$ norms of the input and output,

$$
\|\Gamma(j \omega)\|_{\infty}=\sup _{a_{x}^{\text {target }} \neq 0} \frac{\left\|a_{x}(t)\right\|_{\mathcal{L}_{2}}}{\left\|a_{x}^{\text {target }}(t)\right\|_{\mathcal{L}_{2}}} .
$$

Condition (77) states that the total energy of the output signal is less than than the total energy of the input signal over the interval $t \in[0, \infty)$.

Remark 1: The presented predecessor-follower string stability criterion is more stringent than the leader-follower string stability.

Remark 2: Alternatively string stability can be defined using $\mathcal{L}_{\infty}$, in order to guarantee the absence of overshoot for a desired signal while it propagates throughout the platoon. 


\section{B. Time domain requirements and limitations (longitudinal)}

In addition to the frequency domain requirements introduced in Section III-A, the platoon is subject to limitations and needs to fulfil safety and performance requirements that we formulate in the time domain.

We require that a safe minimum distance is maintained from the preceding vehicle in order to reduce the risk of rear-end collisions. We write this requirement as,

$$
e_{p, \min } \leq e_{p}(t) \leq e_{p, \max }, \quad \forall t \geq 0,
$$

where $e_{p, \max }$ is the maximum allowed distance from the preceding vehicle. While $e_{p, \max }$ can be selected to fulfil a performance criteria (e.g. to not allow increasing the platoon length), the choice of the lower bound in $(8) e_{p, \min }$ is strictly a safety requirement.

Since the primary objective of the automated driving system is to regulate the vehicle velocity to the platoon velocity, we constrain the relative speed between two adjacent vehicles as,

$$
e_{v, \min } \leq e_{v}(t) \leq e_{v, \max }, \quad \forall t \geq 0,
$$

where $e_{v, \min }$ and $e_{v, \max }$ are performance requirements.

We also ensure that all vehicles belonging to the platoon deliver an acceleration/deceleration within desired bounds,

$$
a_{\text {min }} \leq a_{x}(t) \leq a_{\max }, \quad \forall t \geq 0,
$$

where $a_{\min }$ and $a_{\max }$, are the minimum and maximum allowed accelerations, respectively.

To ensure that the acceleration commanded by the controller designed in Section IV is within the admissible actuator (engine and brake) range, the following constraint is introduced

$$
u_{\min } \leq u(t) \leq u_{\max }, \quad \forall t \geq 0 .
$$

We also limit the operating range of the vehicle following application with the following constraint,

$$
0 \leq v_{x}(t) \leq v_{\max }, \quad \forall t \geq 0,
$$

where $v_{\max }$ is the maximum allowed velocity.

We compactly rewrite the constraints $(8)-(12)$ in the following form,

$$
\left[\begin{array}{ll}
H_{\chi} & H_{u}
\end{array}\right]\left[\begin{array}{l}
\chi \\
u
\end{array}\right] \leq h_{\chi} .
$$

\section{Time domain requirements and limitations (lateral)}

Due to physical limitations and in order to ensure safe operation within the lane boundaries, we introduce the following constraints.

In order to formulate constraints on the vehicle position within the lane, we denote by $e_{y_{i j}}, i \in\{f, r\}, j \in\{l, r\}$, the distances of the four vehicle corners from the lane centerline. By assuming small orientation errors, $e_{y_{i j}}$ can be written as

$$
\begin{array}{ll}
e_{y_{f l}}=e_{y}+\frac{c}{2}+a e_{\psi}, & e_{y_{f r}}=e_{y}-\frac{c}{2}+a e_{\psi}, \\
e_{y_{r l}}=e_{y}+\frac{c}{2}-b e_{\psi}, & e_{y_{r r}}=e_{y}-\frac{c}{2}-b e_{\psi},
\end{array}
$$

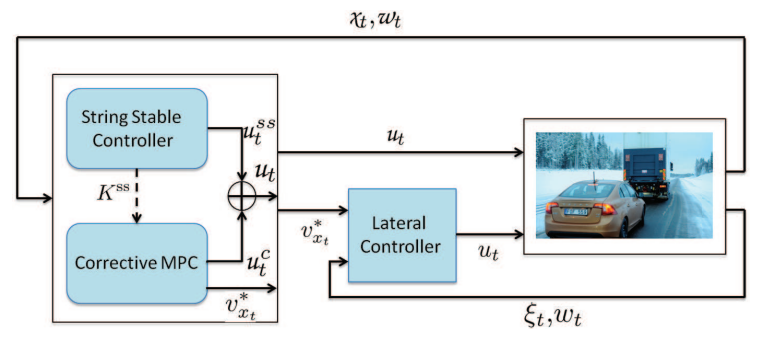

Fig. 2. Control structure block diagram.

where $c$ is the vehicle width, $a$ and $b$ are the distances of the center of gravity from the front and rear vehicle bumpers, respectively.

Furthermore, in order to avoid possible vehicle instability due to the effects of the tire nonlinearities, the vehicle can be forced to operate in a region of the state space by limiting the tire slip angles $\alpha_{i}, i \in\{f, r\}$.

The constraints on the vehicle position and slip angles can then be compactly written as

$$
\begin{aligned}
-e_{y_{\max }} & \leq e_{y_{i j}} \leq e_{y_{\max }}, \\
\alpha_{i_{\min }} & \leq \alpha_{i} \leq \alpha_{i_{\max }}, i \in\{f, r\}, j \in\{l, r\},
\end{aligned}
$$

where $e_{y_{\max }}$ is the maximum distance of the vehicle corners from the lane centerline.

Moreover, the steering angle $u$ in (5) is limited by introducing the following constraints

$$
-\delta_{\max } \leq u \leq \delta_{\max } .
$$

We compactly rewrite the constraints (15) (16) as,

$$
\left[\begin{array}{ll}
H_{\xi} & H_{u}
\end{array}\right]\left[\begin{array}{l}
\xi \\
u
\end{array}\right] \leq h_{\xi} .
$$

\section{Control Design}

In this section, we design controllers for the longitudinal and lateral vehicle motion. A block diagram of the adopted control structure is shown in Figure 2. We divide the control problem into subproblems and design three interconnected controllers to solve each subproblem. In Section IV-A we design a longitudinal headway controller in the frequency domain that ensures string stability of the vehicle platoon. In Section IV-B, we design a corrective controller that modifies the longitudinal control signal $u$ such that the time domain constraints (13) are never violated. Finally, in IV-C we present a lateral controller that accounts for the velocity changes induced by the longitudinal controllers and maintains the vehicle within its designated lane.

\section{A. String stable longitudinal control}

Consider the feedback and feedforward control policy,

$$
u^{s s}=K^{s s}\left[\begin{array}{c}
\chi \\
w
\end{array}\right]=\left[\begin{array}{ll}
K_{\mathrm{FB}}^{s s} & K_{\mathrm{FF}}^{s s}
\end{array}\right]\left[\begin{array}{c}
\chi \\
w
\end{array}\right],
$$

where $K_{\mathrm{FB}}^{s s}$ and $K_{\mathrm{FF}}^{s s}$ are static state feedback and feedforward gains, respectively. Utilizing the control policy $(18)$ to 
generate the control signal for the system (4), i.e. setting $u=u^{s s}$, yields the closed loop system,

$$
\dot{\chi}(t)=A_{\chi}^{\mathrm{cl}} \chi(t)+E_{w}^{\mathrm{cl}} w(t),
$$

where $A_{\chi}^{\mathrm{cl}}=A_{\chi}+B_{u} K_{\mathrm{FB}}^{s s}$ and $E_{w}^{\mathrm{cl}}=B_{u} K_{\mathrm{FF}}^{s s}+E_{w}$. We consider the following output signals,

$$
\left[\begin{array}{c}
a_{x} \\
e_{p} \\
u^{s s}
\end{array}\right]=\left[\begin{array}{cc}
C_{\Gamma} & D_{\Gamma} \\
C_{H} & D_{H}
\end{array}\right]\left[\begin{array}{c}
\chi \\
w
\end{array}\right],
$$

and denote by, $\Gamma=C_{\Gamma}\left(s I-A_{\chi}^{\mathrm{cl}}\right)^{-1} E_{w}^{\mathrm{cl}}+D_{\Gamma}$, the transferfunction from $w \rightarrow a_{x}$ and by $H=C_{H}\left(s I-A_{\chi}^{\mathrm{cl}}\right)^{-1} E_{w}^{\mathrm{cl}}+$ $D_{H}$, the transfer function from $w \rightarrow\left[\begin{array}{ll}e_{p} & u^{s s}\end{array}\right]^{T}$.

Naturally, we want to choose the gain $K^{s s}$ in (18) such that the position error $e_{p}$ stays small, i.e. the controller tracks the desired distance well. In addition we would like to keep the control effort as small as possible. In the frequency domain we thus want to chose $K^{s s}$ such that, the 2-norm, $\|H\|_{2}$ is kept as small as possible.

We also want to choose $K^{s s}$ such that acceleration signals are attenuated as much as possible along the tail of the platoon. We thus want to optimize $K^{s s}$ such that $\|\Gamma\|_{\infty}$ is as small as possible. In particular the condition (6) may not be violated in order to ensure string stability. We calculate $K^{s s}$ as,

$$
\begin{array}{rr}
K^{s s}=\underset{K^{s s}}{\operatorname{argmin}} & \alpha\|\Gamma\|_{\infty}+\beta\|H\|_{2}, \\
\text { subj.to } & \|\Gamma\|_{\infty} \leq 1,
\end{array}
$$

where $\alpha$ and $\beta$ are non-negative tuning parameters that can be chosen to trade off between the different control objectives. The optimization problem (21) can be formulated as an LMI which can be solved using convex optimization [7].

\section{B. Corrective constraint satisfier}

In Section III-A, we designed the feedback/feedforward control law (18) in a frequency domain setting which enabled us to smoothly integrate the string stability criteria (6). However, the integration of the time domain constraints (13) is not a trivial task in the frequency domain. In this section, we integrate the constraints (13) using time domain methods.

Consider the corrective control signal $u^{c}$ and let $u=u^{s s}+u^{c}$. The system (4) can then be written as,

$$
\dot{\chi}(t)=A_{\chi}^{\mathrm{cl}} \chi(t)+B_{u} u^{c}(t)+E_{w}^{\mathrm{cl}} w(t) .
$$

We discretize the model 22 with a sampling time $T_{s}$ and form the discrete time model,

$$
\chi(t+1)=\tilde{A}_{\chi} \chi(t)+\tilde{B}_{u} u^{c}(t)+\tilde{E}_{w} w(t),
$$

where with an abuse of notation we have used the same symbols to denote the time, state, control and disturbance variables.
We formulate the following optimization problem,

$$
\begin{aligned}
& \min _{\mathcal{U}_{t}, \epsilon} \sum_{k=0}^{N-1}\left\|u_{t+k, t}^{c}\right\|^{2}+\gamma \epsilon, \\
& \text { s.t. } \chi_{t+k+1, t}=\tilde{A}_{\chi} \chi_{t+k, t}+\tilde{B}_{u} u_{t+k, t}^{c}+\tilde{E}_{w} w_{t+k, t}, \\
& \\
& {\left[\begin{array}{ll}
H_{\chi} & H_{u}
\end{array}\right]\left[\begin{array}{l}
\chi_{t+k, t} \\
u_{t+k, t}
\end{array}\right] \leq h_{\chi}+\epsilon, } \\
& k=[0, \ldots, N-1], \\
& \quad \epsilon \geq 0, \\
& \quad \chi_{t, t}=\chi(t),
\end{aligned}
$$

where $t$ denotes the current time instant and $x_{t+k, t}$ denotes the predicted state at time $t+k$ obtained by applying the control sequence $\left[u_{t, t}^{c}, \ldots, u_{t+k, t}^{c}\right]$ to the system 23 with $\chi_{t, t}=\chi(t), \mathcal{U}_{t}=\left[u_{t, t}^{c}, \ldots, u_{t+N-1, t}^{c}\right]$ and $N$ denotes the prediction horizon. The constraints (13) have been imposed as soft constraints to ensure feasibility of the optimization problem by introducing the slack variable $\epsilon, \gamma$ is a positive weight penalizing the violation of the soft constraints.

At each time step, we solve the optimization problem (24) using updated information about the states and disturbances and let $u^{c}(t)=u_{t, t}^{c *}$. We remark that no penalty on deviation from a tracking reference is imposed in the cost function 24a). The objective here is to ensure that the constraints (13) are not violated, while utilizing minimal corrective control action $u^{c}$. If the control signal, computed through the control policy (18) is alone capable of controlling the vehicle without violating the constraints (15), no corrective control action will be applied and the optimal cost will thus be zero.

\section{Lateral Control}

Since we have formulated all our lateral requirements and constraints in the time domain we formulate the lateral control problem as an MPC problem. We recall that the lateral vehicle dynamics can be described by the nonlinear model obtained by Equations (1a)-(1e). In Section III-B. we exclude $1 \mathrm{e}$ and consider the longitudinal velocity $v_{x}$ as a parameter of the linear model (5). This allows for utilizing fast convex optimization methods that guarantee global optimality [8]. As shown in Figure 3, the longitudinal velocity $v_{x}$ influences stability of the lateral vehicle dynamics. Disregarding potential future changes in $v_{x}$, as is often done in the literature, e.g. [11], might therefore be risky.

Let $\mathcal{U}_{t}^{*}$ be the optimal control sequence obtained by solving the optimal control problem (24) and denote by $\left[v_{x_{t, t}}^{*}, \ldots, v_{x_{t+N-1, t}}^{*}\right]$ the optimal longitudinal velocity trajectory obtained by applying the control sequence $\mathcal{U}_{t}^{*}$ to the system 23) with $x_{t, t}=x(t)$. We account for predicted future changes in $v_{x}$ by considering the linear discrete time models,

$$
\xi(t+1)=\tilde{A}_{\xi}\left(v_{x_{t, t+k}}^{*}\right) \xi(t)+\tilde{B}_{u} u(t)+\tilde{E}_{w} w(t),
$$

$k=[0, \ldots, N-1]$, formed by discretizing the continuous time lateral vehicle model (5) with $\rho=v_{x_{t, t+k}}^{*}$. Note that we have again abused notation using overlapping symbols with the continuous time model (5). 

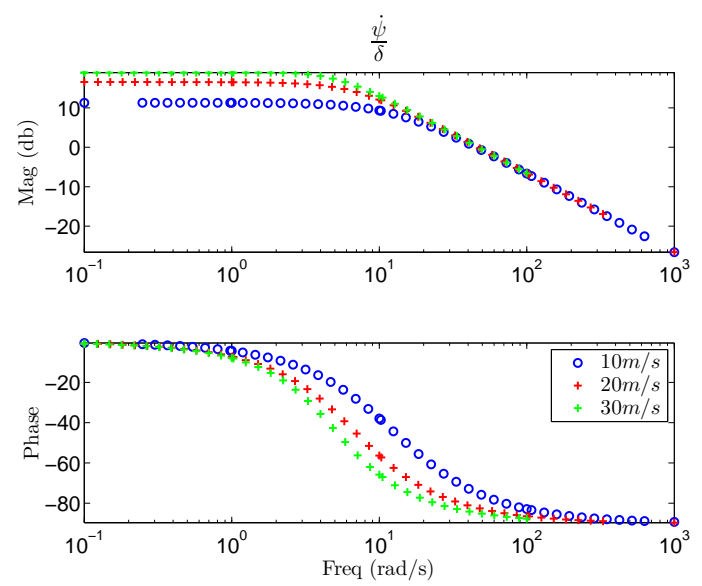

Fig. 3. Frequency response. As can be seen for higher velocity there is a less damping. This can results in an oscillatory control

We let, $\eta=C \xi=\left[v_{y}, e_{\psi}, e_{y}\right]^{T}$, and formulate the following optimization problem,

$$
\begin{aligned}
\min _{\mathcal{V}_{t}, \varepsilon} & \sum_{k=0}^{N-1}\left\|\eta_{t+k, t}\right\|_{Q}^{2}+\left\|u_{t+k, t}\right\|_{R}^{2}+\varrho \varepsilon, \\
\text { s.t. } & \xi_{t+k+1, t}=\tilde{A}_{\xi} \xi_{t+k, t}+\tilde{B}_{u} u_{t+k, t}+\tilde{E}_{w} w_{t+k, t}, \\
& \eta_{t+k, t}=C \xi_{t+k, t}, \\
& {\left[\begin{array}{ll}
H_{\xi} & H_{u}
\end{array}\right]\left[\begin{array}{l}
\xi_{t+k, t} \\
u_{t+k, t}
\end{array}\right] \leq h_{\xi}+\varepsilon, } \\
& k=[0, \ldots, N-1], \\
& \varepsilon \geq 0, \\
& \xi_{t, t}=\xi(t),
\end{aligned}
$$

where $\mathcal{V}_{t}=\left[u_{t, t}, \ldots, u_{t+N-1, t}\right], \varepsilon$ is a slack variable and $Q, R, \varrho$ are positive definite weights on the states, control and slack variable, respectively. We solve 26 at each time instant and let $u(t)=u_{t, t}^{*}$.

We remark that while the lateral controller adjusts steering to account for speed variations induced by the longitudinal control, it does not adjust the speed. In the platooning application, the lead vehicle is expected to handle necessary speed adjustments due to e.g. changing road curvature.

\section{RESUlts}

In the following subsections we evaluate the performance of the proposed control approaches.

\section{A. Longitudinal tracking performance and string stability}

Consider the trajectories shown in Fig. 4. The trajectories have been generated by simulating a platoon consisting of three vehicles, i.e., one leader and two followers. The two followers are controlled using the control approaches suggested in Section IV. The leader starts by cruising at a constant speed of $7 \mathrm{~m} / \mathrm{s}$, makes two harsh accelerations to $20 \mathrm{~m} / \mathrm{s}$ and $30 \mathrm{~m} / \mathrm{s}$, respectively, and then two consecutive harsh brakings. In Fig. 4 we note that the two followers perform well in tracking their respective position and velocity references. By studying the acceleration profiles shown in
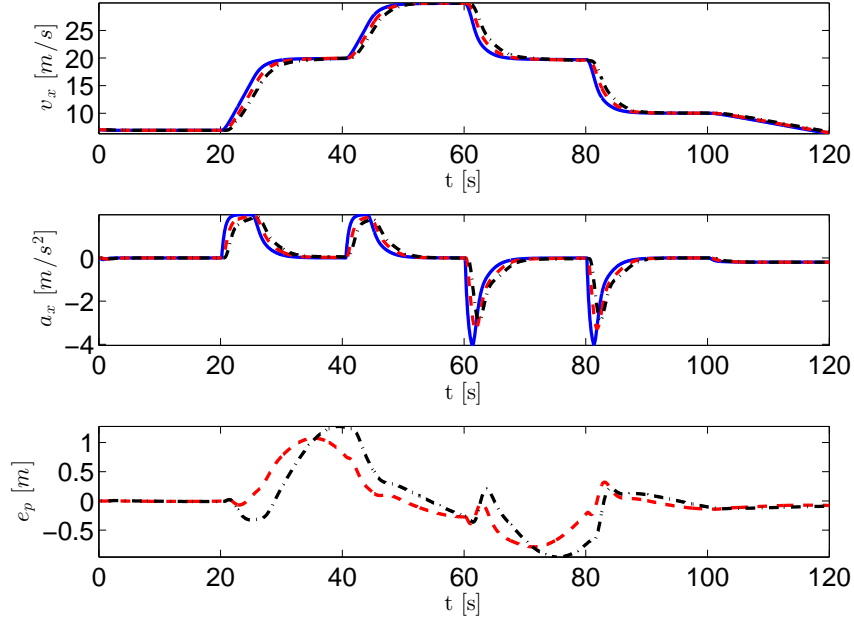

Fig. 4. From top to bottom, velocity profiles, acceleration signals and position errors between the adjacent vehicles, respectively. Velocities and accelerations of leader, Follower 1 and Follower 2 are in solid blue, dashed red and dashed dotted black, respectively. Position errors of Followers 1 and 2 in dashed red and dashed dotted black, respectiveley.

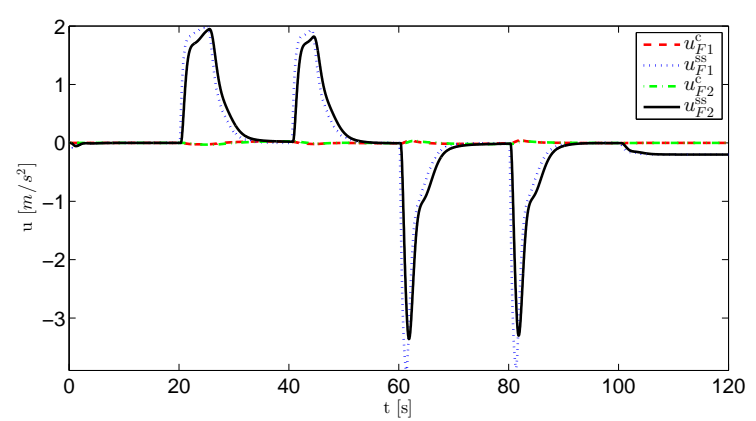

Fig. 5. Control signals, $u^{s s}$ and $u^{c}$ computed in the two follower vehicles, named as F1 and F2, respectively. We note that, in the considered scenario, the string stable controllers do not violate any of the constraints since no corrective control action $u^{c}$ is needed in any of the vehicles.

Fig. 4 we also note that the longitudinal accelerations are damped out throughout the platoon such that the string stability criterion (7) is fulfilled throughout the trajectory.

The control signals $u^{c}$ and $u^{s s}$, computed in the two follower vehicles are shown in Fig. 5. In the simulated scenario, the contribution of the string stable controller $u^{s s}$, is alone capable of maintaining the vehicle state within the constraints (13) and there is therefore no no need for corrective control action $u^{c}$.

\section{B. Longitudinal constraint satisfaction}

While Section $\mathrm{V}-\mathrm{A}$ was devoted to demonstrating performance and string stability, we demonstrate the ability of the suggested approach to maintain the vehicle state within the constraints (13) in this section. Consider the trajectories shown in Fig. 6 The trajectories have been collected in a prototype follower (Volvo S60) vehicle while following a 

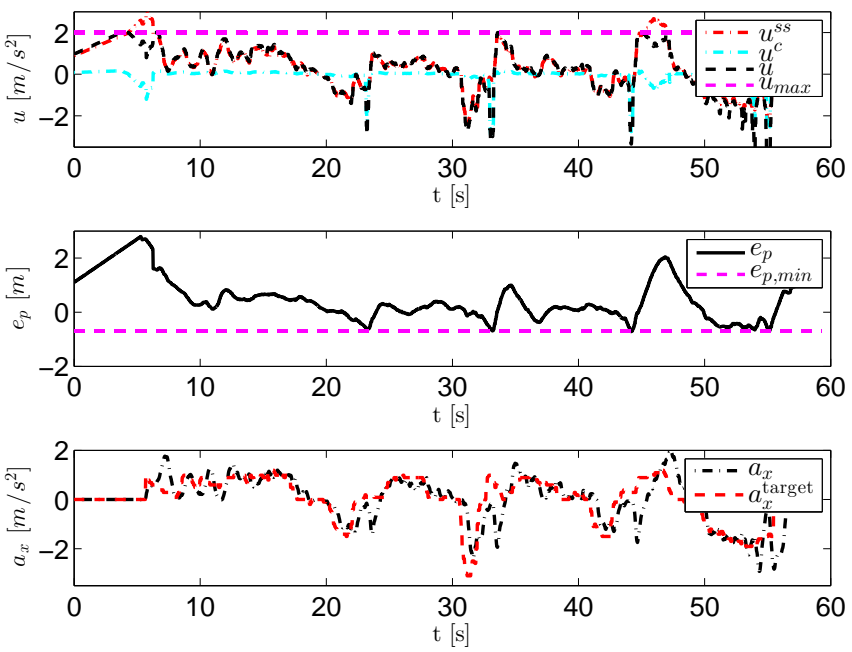

Fig. 6. To the top, the control command of string stable controller, corrective controller and the sum of both are presented in dashed dotted red, dashed dotted blue and dashed black, respectively. The dashed magenta shows the upper constraint on the control signal. In the middle, the position error and the lower constraint on the position error are represented in solid black and dashed magenta, respectively. To the bottom the acceleration of the target vehicle and follower are shown.

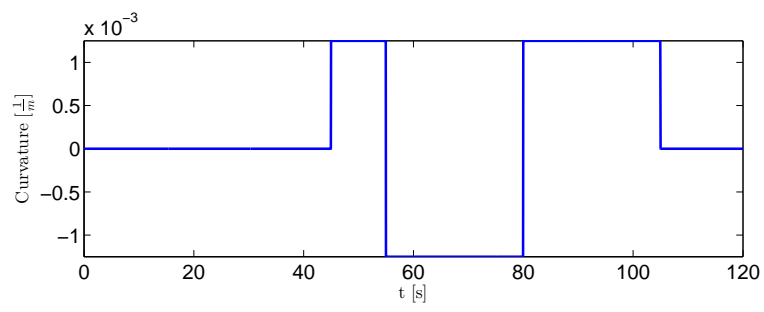

Fig. 7. Road curvature

leader (Volvo S80). The follower vehicle is equipped with wireless communication and a rapid prototyping system in which the suggested control approach has been implemented.

We have made the task of satisfying the constraints (13) difficult for the string stable controller (18) through the bounds $e_{p, \min }=-0.6 \mathrm{~m}$ and $u_{\max }=2 \frac{\mathrm{m}}{\mathrm{s}^{2}}$. Fig. 6 reveals that, at time $t=6 \mathrm{~s}, u^{s s}$ exceeds $u_{\max }$, and that, at times $t=23,33,43 \mathrm{~s}, e_{p}$ hits the constraint -0.6 . Fig. 6 also shows that the signal $u^{c}$ takes a non-zero value at the same time instances, while $u^{c}=0$ otherwise. We also remark that the string stability criteria (7) is satisfied as long as $u^{c}=0$ while it is relaxed when necessary in order to satisfy the constraints (13).

\section{Lateral control performance}

In this section we evaluate the performance of the suggested lateral control approach. Let us again consider the trajectories shown in Fig. 4. The curvature of the road traversed by the vehicle platoon in the considered simulation is shown in Fig. 7

The states of the lateral dynamics for follower 1 are depicted in Fig. 8. Clearly, the suggested LPV-MPC controller
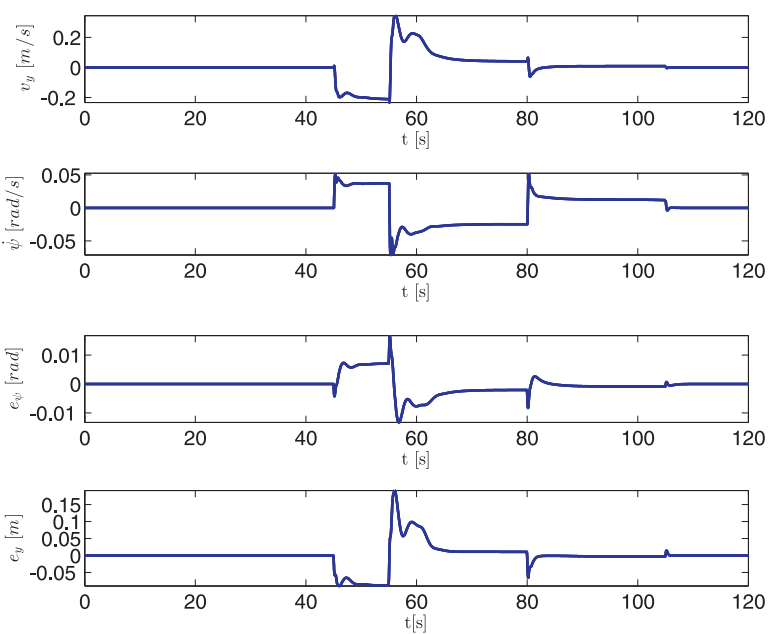

Fig. 8. Lateral states of vehicle, the lateral velocity, yaw rate, yaw error and lateral position error from top to the bottom, respectively.

is capable of keeping the vehicle within the lane with small tracking errors in spite of the rather big variation in $v_{x}$. The constraints are set as, $e_{y_{\max }}=1.5 \mathrm{~m}, e_{y_{\min }}=-1.5 \mathrm{~m}$, $\alpha_{\min }=-4^{\circ}, \alpha_{\max }=4^{\circ}, \delta_{\min }=-45^{\circ}$ and $\delta_{\max }=45^{\circ}$.

\section{CONCLUDING REMARKS}

We have combined frequency and time domain methods to design a safe, string stable control approach. The limited results presented in this paper indicate that the suggested approach can be successfully used for controlling a vehicle platoon while fulfilling a wide range of requirements.

\section{ACKNOWLEDGMENTS}

The research leading to these results has received funding from the European Commission Seventh Framework Programme under the project AdaptIVe, grant agreement number 610428. The author(s) would like to thank all partners within AdaptIVe for their cooperation and valuable contribution.

\section{REFERENCES}

[1] P. Barooah and J. Hespanha. Error amplification and disturbance propagation in vehicle strings with decentralized linear control. In 44th IEEE Conference on Decision and Control, Boston, Massachusetts, USA, December 2005.

[2] E. Coelingh and S. Solyom. All Aboard the Robotic Road Train. IEEE Spectrum, pages 34-39, 2012.

[3] W.B. Dunbar and D.S. Caveney. Distributed receding horizon control of vehicle platoons: Stability and string stability. Automatic Control, IEEE Transactions on, 57(3):620 -633, march 2012.

[4] R. Kianfar, B. Augusto, A. Ebadighajari, U. Hakeem, J. Nilsson, A. Raza, R. S. Tabar, N. V. Irukulapati, C. Englund, P. Falcone, S. Papanastasiou, L. Svensson, and H. Wymeersch. Design and experimental validation of a cooperative driving system in the grand cooperative driving challenge. Intelligent Transportation Systems, IEEE Transactions on, 13(3):994-1007, sept. 2012.

[5] Roozbeh Kianfar, Paolo Falcone, and Jonas Fredriksson. A distributed model predictive control approach to active steering control of string stable cooperative vehicle platoon. In Advances in Automotive Control, volume 7, 2013.

[6] Shengbo Li, Keqiang Li, Rajesh Rajamani, and Jianqiang Wang. Model predictive multi-objective vehicular adaptive cruise control Control Systems Technology, IEEE Transactions on, 19(3):556-566, 2011. 
[7] Jan P. Maschuw, Günter C. Keßler, and D. Abel. LMI-based Control of Vehicle Platoons for Robust Longitudinal Guidance. In 17th IFAC World Congress, Seoul, Korea, July 2008.

[8] David Q Mayne, James B Rawlings, Christopher V Rao, and Pierre OM Scokaert. Constrained model predictive control: Stability and optimality. Automatica, 36(6):789-814, 2000.

[9] G.J.L. Naus, Rene P.A . Vugts, J. Ploeg, J.G de Molengraft, and M. Steinbuch. String-stable CACC design and experimental validation : A frequency-domain approach. IEEE Transaction on vehicular technology, 59(9):4268-4279, 2010.

[10] PATH. California partners for advanced transportation technology (PATH). http://www.path.berkeley.edu 1986.

[11] R. Rajamani. Vehicle Dynamics and Control. Springer, 2005.

[12] P. Seiler, A. Pant, and K. Hedrick. Disturbance propagation in vehicle strings. IEEE Transaction On Automatic Control, 49(10):1835-1841, 2004. 